\title{
Radiopaque markers and hydrogram in feline alimentary lymphoma
}

\author{
Jihye Choi ${ }^{1, *}$, Jinsoo Lee ${ }^{2}$, Jaeyoung Jang², Heeyeon Choi ${ }^{2}$, Jimin $\mathrm{Seo}^{2}$, Minjung Lee ${ }^{2}$, Hyunwook Kim² \\ ${ }^{1}$ Department of Veterinary Medical Imaging, College of Veterinary Medicine, Chonnam National University, \\ Gwangju 500-757, Korea \\ ${ }^{2}$ Haemaru Referral Animal Hospital, Seongnam 463-050, Korea \\ (Received: February 2, 2012; Revised: June 6, 2012; Accepted: June 8, 2012)
}

\begin{abstract}
Radiography, ultrasonography with hydrogram, and contrast studies using radiopaque markers were applied to evaluate alimentary lymphoma in two cats. The hydrogram facilitated the differentiation of pseudo-thickening from true wall thickening, and enabled an evaluation of wall layering and lymph nodes. In case 1, mechanical obstruction of the duodenum was confirmed with barium-impregnated polyethylene spheres (BIPS), a radiopaque marker; however, results obtained in case 2 were not as definitive. We expect that hydrograms and BIPS can be used as valuable alternative methods to evaluate the gastrointestinal (GI) tract although further studies in cases involving GI tumors are needed.
\end{abstract}

Keywords : alimentary lymphoma, barium-impregnated polyethylene spheres, cat, hydrogram, ultrasonography

Lymphomas are the most common gastrointestinal (GI) tumors of cats, and endoscopy, radiography, ultrasonography, and contrast studies are usually applied in instances of GI tumors [1, 3, 8]. The argument against the use of endoscopy in the diagnosis of GI lymphoma includes the fact that this technique procures specimens from superficial mucosal layers, potentially missing lymphomas located deeper in the tissue. Although in cats the incidence of deep intestinal lymphomas alone is unknown, they may occur in some cases [2]. Radiographic findings for GI tumors are generally nonspecific, unless mechanical obstruction caused by the neoplasia occurs [7]. Although ultrasonographic findings of lymphoma are not specific, thickening of the gastric or intestinal wall is the most common abnormality found with ultrasonography in GI tumors [3, 7]. Loss of normal intestinal wall layering, a localized mass associated with the GI tract, decreased wall echogenicity, localized hypomotility, regional lymphadenopathy, and ascites are also commonly seen $[3,5,7]$. Radiopaque markers are the technique of choice for quantitative assessing gastric emptying and small and large intestinal transit times and for ruling out partial bowel obstructions, because of their convenience, cost-effectiveness, and established diagnostic accuracy [4, 10]. Barium-impregnated polyethylene spheres (BIPS) are radiopaque markers using two sizes of spheres, $1.5 \mathrm{~mm}$ and $5.0 \mathrm{~mm}$ in diameter and has been known as the most preferable radiopaque markers in veterinary medicine $[4,6]$.

This report describes two cats with alimentary lymphoma diagnosed particularly using hydrogram and contrast studies using BIPS to demonstrate intestinal mechanical obstruction with minimal ultrasonographic changes in these alimentary lymphoma cases.

A 12-year-old, spayed, female mixed-breed cat (case 1) presented with chronic vomiting, weight loss, and depression for six months. Abdominal radiography showed gastric distension and an unusual gas silhouette in the descending duodenum (Figs. 1A and B). On ultrasonography, the stomach and middle region of the ascending duodenum were severely dilated and swirling of hyperechoic fluid was observed within the lumen (Fig. 2). Distal to the middle region of the ascending duodenum, the intestinal lumen returned to a normal diameter. Foreign bodies or marked tumoral lesions causing an obstruction of the intestine were not identified. Obvious peristalsis was found in the dilated region of the stomach and duodenum; however, GI motility did

\footnotetext{
*Corresponding author

Tel: +82-62-530-2821, Fax: +82-62-530-2809

E-mail: imsono@chonnam.ac.kr
} 

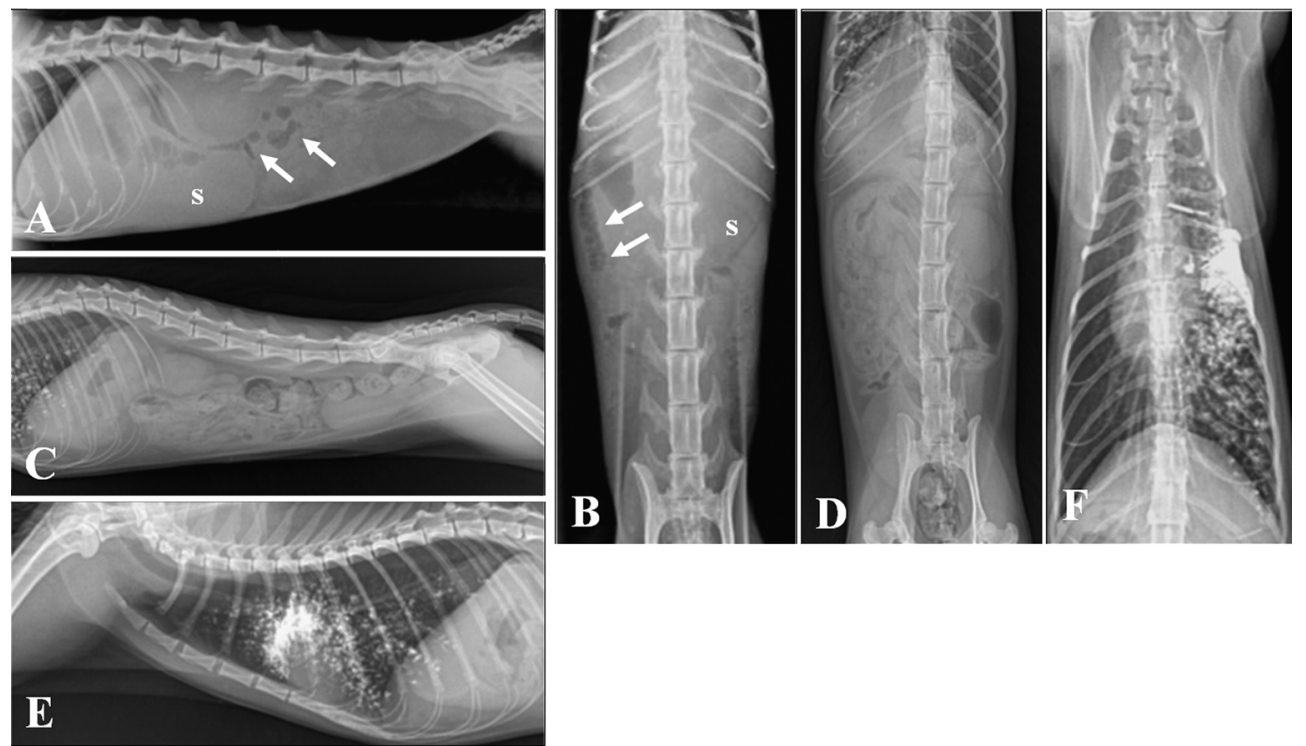

Fig. 1. Radiography. (A, B) In case 1, note the gastric distension (s) and unusual gas formation (arrows) in the descending duodenum. (C-F) In case 2, no remarkable findings except for radiopaque spots in the thoracic region considered to be aspirated barium from a previous upper gastrointestinal contrast study.

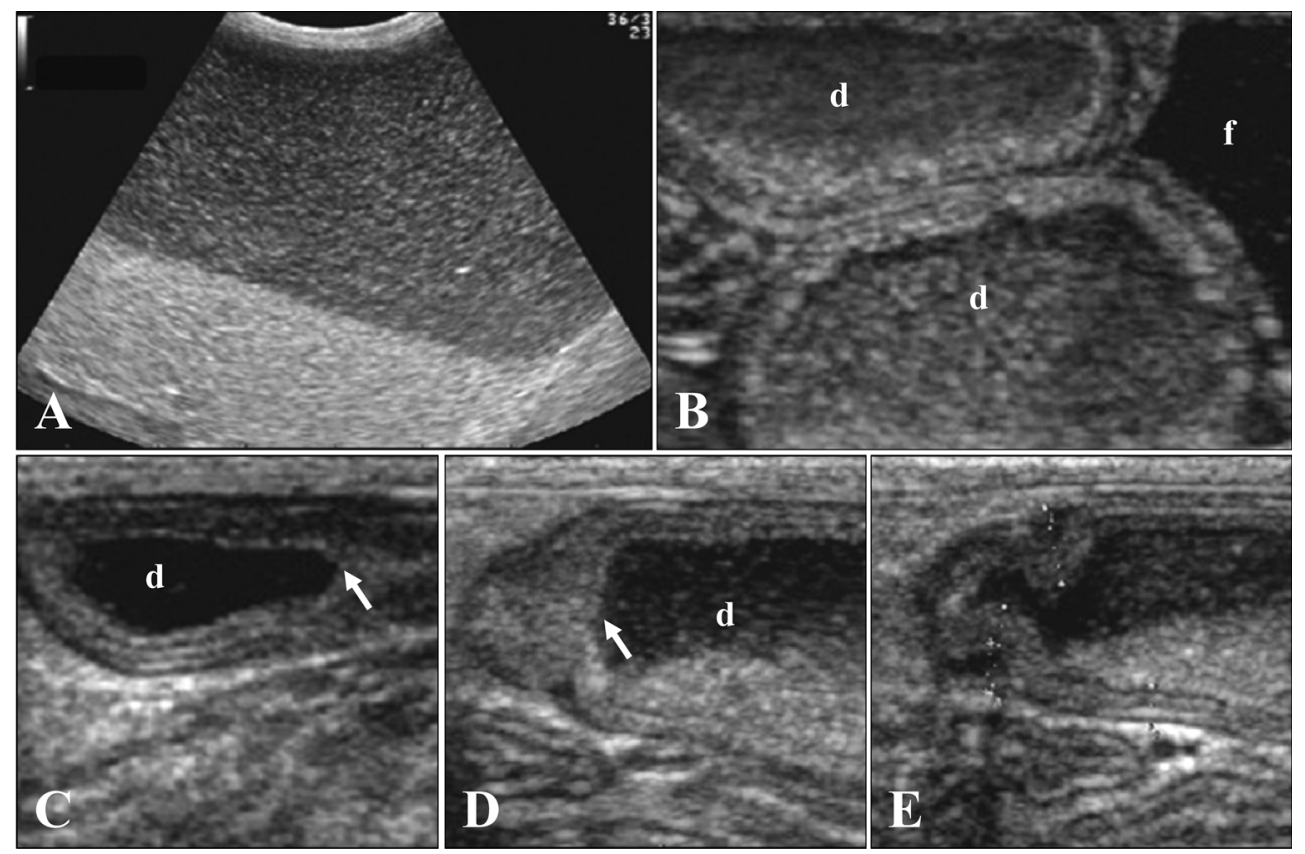

Fig. 2. Ultrasonography of case 1. (A, B) Stomach and middle region of the ascending duodenum (d) are severely dilated with swirling of hyperechoic fluid. (C, D) After the middle region of the ascending duodenum (d), the intestinal lumen returns to normal diameter (arrow). (E) Note the normal duodenal wall thickness and wall layering.

not continue past the distal area of the dilated portion of the ascending duodenum, and the lumen at this point of the duodenum was not open. Marked ultrasonographic changes such as a thickened intestinal walls or 
loss of wall layering were not observed. Anechoic abdominal fluid and mild enlargement of the mesentery lymph nodes were found. In BIPS (MedID, USA) study taken radiographs at $3 \mathrm{~h}$ intervals for $15 \mathrm{~h}$, most BIPS were vomited with vigorous salivation except for 6 of 10 large spheres and 5 of 30 small spheres approximately 10 min after administration of the contrast agent. After that, the remaining large and small spheres were retained in the right middle abdomen (Figs. 4A-D). An intestinal obstruction with intramural lesions was diagnosed.

Upon exploratory laparotomy, the intestinal lumen was found to be narrowed at the junction of the ascending duodenum and jejunum resulting in stagnation of the BIPS. No remarkable wall thickening or mass lesions were observed upon firm palpation of the region. The mesentery lymph nodes were enlarged. An enterostomy was performed to remove the narrowed region and a 5 French red rubber catheter was placed for feeding via a jejunostomy. Oral feeding was restarted 3 days after surgery. The cat was discharged from the hospital 6 days after surgery with a stable body condition and restored appetite. Low grade alimentary lymphoma was diagnosed histopathologically. Chemotherapy was not applied because of the owner's refusal.

A seven-year-old, spayed female, Russian Blue cat (case 2) presented with a history of vomiting once every two or three days for the past five months. The frequency of vomiting had increased over the last two months. Radiographically, multiple radiopaque spots suspected as being aspirated barium used in a GI study by the referring veterinarian were found in the right pulmonary area (Figs. 1C-F). The GI tract could not be evaluated sonographically because of the large amount of gas (Fig. 3). Mesenteric lymph nodes around the stomach were moderately enlarged and showed hypoechoic changes and a round shape. After $12 \mathrm{~h}$ of fasting, the GI tract was rechecked using the fluid-filled stomach method, referred to as hydrogram. This procedure was done with the administration of $40 \mathrm{~mL}$ of fluid through a gastric tube followed immediately by real-time ultrasonography. The gastric wall and lumen could be clearly evaluated using the hydrogram, and no abnormal lesions were observed. A focal thickening of the wall of the proximal duodenum, as much as $5 \mathrm{~mm}$, was observed compared to the adjacent duodenal wall, which was only $2 \mathrm{~mm}$. The thickened duodenal wall was hypoechoic and lacked normal wall layering. The lumen at this point of the duodenum was not open although
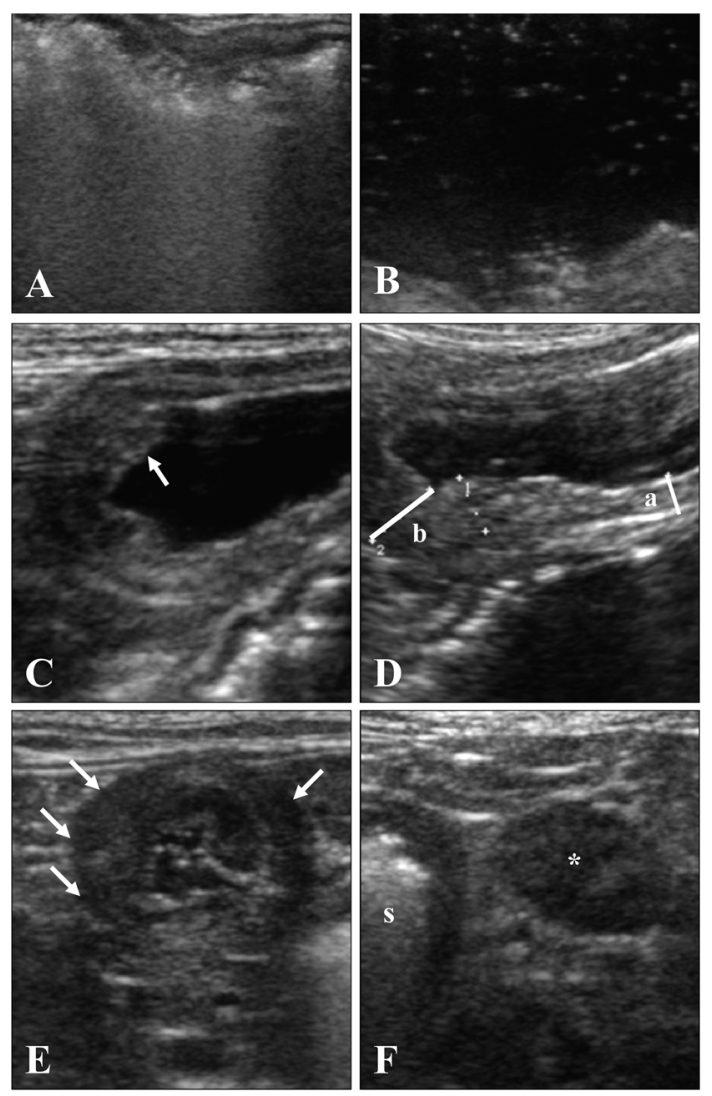

Fig. 3. Ultrasonography of case 2. (A) The stomach has a large amount of gas. (B) The hydrogram clearly shows the gastric wall and lumen. (C, D) Focal thickening of the proximal duodenal wall (arrow) as much as $5 \mathrm{~mm}$ (b), compared to the adjacent $2 \mathrm{~mm}$ duodenal wall (a). (E) The thickened duodenal wall is hypoechoic (long arrow) and loses normal wall layering (short arrows). (F) Enlarged mesenteric lymph nodes $(*)$ are found around the stomach (s).

adjacent intestinal loops showed normal motility. Partial obstruction of the duodenum by intestinal neoplasia or severe infiltrative disease was suspected. In a BIPS study using the same protocol as in case 1, only a large sphere was retained within the stomach and other large and small spheres moved without delayed transit or accumulation in a specific region (Figs. 4E-H).

On laparotomy, the proximal duodenum at the gastroduodenal junction was found to be thickened upon firm palpation. Mesenteric lymph nodes in the vicinity of the stomach were enlarged to approximately $2 \times 0.7$ $\mathrm{cm}$. The duodenal mass and lymph nodes were subsequently removed. The cat recovered from anesthesia 

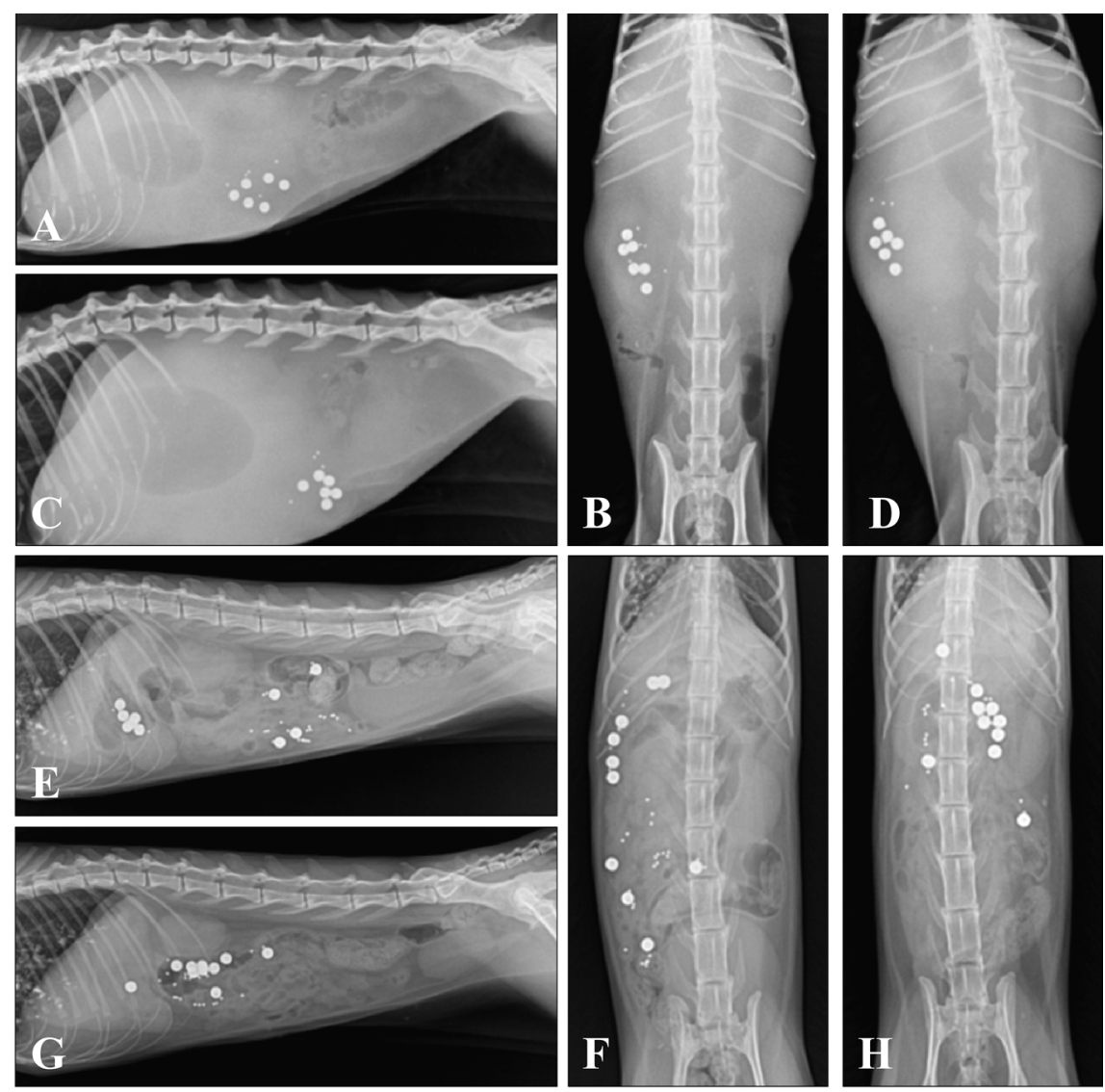

Fig. 4. Contrast study using barium-impregnated polyethylene spheres (BIPS). (A, B) In case 1, only 6 large spheres and 5 small spheres are found following vomiting. The large and small spheres are retained in the duodenal region in the right middle abdomen. (C, D) The retention of radiopaque spheres is not changed after $9 \mathrm{~h}$. (E, F) Three hours after administration of BIPS in case 2, most large and small spheres are eliminated from the stomach. (G, H) In radiographs taken at $9 \mathrm{~h}$ after administration, only one large sphere remains in the stomach and others enter the large intestine.

uneventfully, however on the day following surgery, the cat died after a bout of severe vomiting. Histopathologically, the duodenal mass was diagnosed as intermediate-grade lymphoma extending throughout the submucosa and into portions of the muscular wall. Metastasis to the lymph nodes was confirmed. Immunohistochemical staining and autopsy could not be performed.

Ultrasonography of the GI system is very useful in defining masses originated from GI tract and interrogation of the wall layers. Lymphoma can show thickening of GI wall with or without loss of wall layering, decreased wall echogenicity, and localized hypomotility, or a mass associated with the GI tract [3, $5,7]$. These changes can be evaluated using ultrasono- graphy and play a role to provide useful information for differentiating GI tumors such as lymphoma from inflammatory diseases.

Case 2 had ultrasonographic findings of intestinal wall thickening with loss of normal wall layering and decreased intestinal wall echogenicity and regional lymphadenopathy. However, case 1 showed only localized hypomotility without any anatomical changes of the intestines.

In this report, the lumen and the wall of GI tract were evaluated using "hydrogram", especially the luminal opening of the lesion suspected as mechanical obstruction. The fluid-filled stomach in hydrogram allows visualization of the gastric and duodenal wall and lumen with realtime ultrasonographic examination [6]. This method has 
higher sensitivity to detect tumors of the GI tract compared to fasting conditions, and it may be considered as complementary to endoscopy in the diagnosis of submucosal tumors and as an alternative to other expensive examinations such as endoscopic echography [9]. In case 2, the hydrogram demonstrated a duodenal lesion which was not observed by ultrasonography without fluid. The hydrogram helped to differentiate abnormal wall thickening of the stomach and duodenum from pseudo-thickening, which might occur in fasting conditions, and provided good visualizing of wall layering. Pre-existing gas in the stomach and duodenum might interfere with evaluation of the GI tract even with the hydrogram; however, this disturbance can be minimized by using the relationship between fluid and gas gravity and adjusting the patient's position accordingly.

The diagnostic accuracy of radiopaque markers such as BIPS are high in comparison to studies with barium suspensions. In case 1, mechanical obstruction of the intestine suspected from ultrasonography could be confirmed with BIPS on the basis of retention of large and small spheres. Although in case 1 most spheres were vomited after administration, this did not influence the study results. However, in case 2, most large and small spheres (except for one large sphere) entered the large intestine passing through the upper GI tract without an abnormal transit pattern, despite the marked duodenal lesion revealed by ultrasonography. Inconsistent results as occurred in these two cases and failure to outline the GI mucosa limit the applicability of radiopaque markers.

Results of this study indicate that ultrasonography is an effective noninvasive means of identifying lesions consistent with alimentary lymphoma in cats. In particular, ultrasonography using the fluid-filled stomach method, hydrogram, can provide clear images of the GI tract and underlying organs including mesentery and lymph nodes. Radiopaque marker studies do not replace any currently available diagnostic modalities; rather, they can provide additional information to compliment radiography and ultrasonography results. In this study, BIPS helped to definitively diagnose partial obstruction of the duodenum in case 1 even when anatomical changes related to the GI tumor were not identified by ultrasonography. However, in case 2, which had remarkable intestinal wall thickening and loss of normal wall layering, BIPS provided ambiguous results.

GI tumors can only be definitively confirmed histopathologically; however, ultrasonography with hydrogram and contrast study with radiopaque markers can provide additional helpful information to assist in the correct diagnosis of GI tumors.

\section{References}

1. Arista-Nasr J, Jimenez A, Keirns C, Larraza O, Larriva-Sahd $\mathbf{J}$. The role of the endoscopic biopsy in the diagnosis of gastric lymphoma: a morphologic and immunohistochemical reappraisal. Hum Pathol 1991, 22, 339-348.

2. Couto CG, Rutgers HC, Sherding RG, Rojko J. Gastrointestinal lymphoma in 20 dogs. A retrospective study. J Vet Intern Med 1989, 3, 73-78.

3. Grooters AM, Biller DS, Ward H, Miyabayashi T, Couto CG. Ultrasonographic appearance of feline alimentary lymphoma. Vet Radiol Ultrasound 1994, 35, 468-472.

4. Hall JA. Clinically evaluating gastric diseases in dogs and cats. Vet Med 2000, 95, 450-465.

5. Hittmair K, Krebitz-Gressl E, Kübber-Heiss A, Möstl K. Feline alimentary lymphosarcoma: Radiographical, ultrasonographical, histological and virological findings. Eur J Companion Anim Pract 2001, 11, 119128.

6. Kremer H, Gröbner W. Sonography of polypoid gastric lesions by the fluid-filled stomach method. J Clin Ultrasound 1981, 9, 51-54.

7. Penninck DG, Moore AS, Tidwell AS, Matz ME, Freden GO. Ultrasonography of alimentary lymphosarcoma in the cat. Vet Radiol Ultrasound 1994, 35, 299-304.

8. Roth L, Leib MS, Davenport DJ, Monroe WE. Comparisons between endoscopic and histologic evaluation of the gastrointestinal tract in dogs and cats: 75 cases (1984-1987). J Am Vet Med Assoc 1990, 196, 635-638.

9. Tous F, Busto M. Assessment of abdominal sonography in the diagnosis of tumors of the gastroduodenal tract. J Clin Ultrasound 1997, 25, 243247.

10. Wyse CA, McLellan J, Dickie AM, Sutton DGM, Preston T, Yam PS. A review of methods for assessment of the rate of gastric emptying in the dog and cat: 1898-2002. J Vet Intern Med 2003, 17, 609621. 